\title{
Breast Cancer Stromal Myxoid Changes Are Associated with Tumor Invasion and Metastasis: A Central Role for Hyaluronan
}

\author{
Mario Wernicke, M.D., Laura Cecilia Piñeiro, M.D., Daniela Caramutti, M.D., Vanesa G. Dorn, M.D., \\ Maria Marta Lopez Raffo, M.D., Hector G. Guixa, M.D., Margarita Telenta, M.D., \\ Ana Alcestes Morandi, M.D. \\ Department of Pathology (MW, LCP, DC, VGD, MMLR, MT, AAM) and Breast Cancer Service (HGG), \\ Hospital Italiano, Buenos Aires, Argentina
}

The interplay between a tumor and its environment is exemplified by the morphological changes observed in the stroma of human breast cancer. These changes are evident as stromal myxoid changes. Hyaluronan, an extracellular polysaccharide that has been implicated in invasion, is one of the major constituents of the stromal myxoid changes. This study evaluated the association of these stromal changes with axillary node status, tumor grade, and mortality. The prognostic value of the stromal myxoid changes was evaluated in patients with negative axillary nodes with 10 years of follow-up. Our results showed a high level of reproducibility of our stromal myxoid changes grading system (overall kappa $=0.68$ ). Image analysis semiquantification showed marked correlation of a strong stromal hyaluronan signal with high-grade stromal myxoid changes. In a multiple logistic regression analysis, positive nodes were associated with stromal myxoid changes, tumor size, desmoplasia, lymphocytic infiltration, high tumor grade, tumor emboli, and multifocality. Stromal myxoid changes were also associated with young age and lymphatic embolizations $(P<.001)$. Overall, there is a weak correlation between mortality and stromal myxoid changes $(P$ $<.01)$. Mortality was more evident with high stromal myxoid changes grades and tumor size $>2 \mathrm{~cm}$ $(P<.008)$. However Cox multivariate analysis fail to show stromal myxoid changes as an independent prognostic factor. In conclusion, stromal myxoid changes with high hyaluronan concentration are strongly associated with positive nodes, tumor

Copyright $(\odot 2003$ by The United States and Canadian Academy of Pathology, Inc.

VOL. 16, NO. 2, P. 99, 2003 Printed in the U.S.A.

Date of acceptance: November 15, 2002.

Address reprint requests to: Mario Wernicke, M.D., Hospital Italiano,

Gascon 450, Buenos Aires (1181), Argentina; fax: 5411-4959-0352; e-mail: mariowernicke@hotmail.com.

DOI: 10.1097/01.MP.0000051582.75890.2D grade, and lymphatic emboli, thereby identifying high-risk group and reinforcing the role of hyaluronan in invasion and metastasis.

KEY WORDS: Breast cancer, Hyaluronan, Metastasis, Stroma.

Mod Pathol 2003;16(2):99-107

Lymph node and hematogenous distant metastases are the result of a cascade of enzymatic interactions. Part of this complex cascade takes place in the tumor environment, where malignant tumor cells detach from the primary tumor, adhere to components of the extracellular matrix, degrade them, and move through the stroma. Morphological and biochemical modifications of the different stromal components (cellular and noncellular) can either favor or inhibit this process. Therefore, the histopathological modifications of the stroma in response to a tumor could shed light on the biological mechanisms involved in invasion and metastasis.

Stromal myxoid changes consists of a stromal reaction composed of an amphophilic or slightly basophilic vacuolated material that stains positively with Alcian Blue and is found among the collagen fibers. Hyaluronan is one of its major components. Tissues rich in hyaluronan entrap water and swell, leading to myxoid changes. It is known that hyaluronan synthesis is stimulated by the interaction between tumor and stromal cells (1-3). Hyaluronan has several physiological functions, such as water homeostasis and regulation of capillary growth; it also plays a role in cell recognition and migration (4). A strong relationship between hyaluronan and the metastatic process has been reported in basic oncology research (5-8) as well as in clinical studies (9-14). Toole and coworkers (5) have suggested that because of its unique hydrodynamic properties, 
hyaluronan may open spaces in the connective tissue, allowing tumor migration.

In this study, we correlated stromal myxoid changes with the presence or absence of axillary metastasis, tumor grade, size, and age using multivariate and cross tabulation analysis, as well as stromal myxoid changes prognostic assessment in a cohort of negative axillary nodes.

\section{MATERIAL AND METHODS}

\section{Selection of Cases}

\section{First Group}

To find whether there exists a correlation between weak and strong stromal signal with visual assessment of stromal myxoid changes, 46 cases were selected for staining with a hyaluronan binding biotinylated probe, as will be explained below.

\section{Second Group}

With the sole purpose of testing stromal myxoid changes reproducibility, 202 consecutive cases were evaluated. The level of agreement among three independent observers (LCP, MLR, AAM) for this variable was evaluated with kappa statistics. The kappa statistical measure of agreement is scaled to be 0 when the level of agreement is what would be expected to be observed by chance and to be 1 when there is perfect agreement. For intermediate values, the following interpretation was used: poor: $0.00-0.20$, slight: $0.21-0.40$, moderate: $0.41-$ 0.60 , substantial: $0.61-0.80$, and almost perfect: 0.81-0.99 (15).

\section{Third Group}

A total of 1717 consecutive cases from the period between 1987 and 1999 were retrieved from the files of our pathology laboratory. Cases without axillary sampling or having neoadjuvant chemotherapy were discarded from the study, leaving a total of 1119 cases for evaluation. None of the patients had preoperatory radiotherapy ruling out the possibility of changes caused by treatment. The clinicopathological characteristics of the patients are listed in Table 1.

\section{Fourth Group}

Three hundred one cases of negative axillary nodes were evaluated for prognostic assessment. Time of death was entered as a dependent variable, and it was listed according to the year of the event. The cause of death was obtained from the patients' records. Fifty-four patients with high-grade tumors were selected for adjuvant chemotherapy (16). Intraductal carcinomas were discarded from the study. Mucoid carcinomas were also ruled out because the presence of mucoid material is tumor
TABLE 1. Third Group; Clinicopathological Characteristics

\begin{tabular}{ccc}
\hline Variables & Count & Percent \\
\hline Lymphatic & & \\
0 & 994 & 88.8 \\
1 & 125 & 11.1 \\
SMC & & \\
1 & 469 & 41.9 \\
2 & 351 & 31.3 \\
3 & 299 & 26.7 \\
TG & & \\
1 & 106 & 9.4 \\
2 & 703 & 62.9 \\
3 & 310 & 27.6 \\
Age & & \\
$<45$ & 184 & 16.8 \\
$45-65$ & 507 & 45.3 \\
$>66$ & 289 & 27.7 \\
Missing & 114 & 10.7 \\
Size & & \\
1 & 168 & 15.0 \\
2 & 469 & 41.9 \\
3 & 472 & 42.1 \\
Missing & 10 & 0.9 \\
TIL & & \\
1 & 815 & 72.8 \\
2 & 221 & 7.4 \\
3 & 83 & 58.7 \\
ALN & & 41.2 \\
0 & 657 & \\
1 & 462 & \\
\hline & & \\
\hline
\end{tabular}

secretion, not a stromal reaction. Clinical pathological data for this group are listed in Table 2.

\section{Stromal Myxoid Changes}

Stromal myxoid changes can be defined as an amorphous stromal substance composed of an amphophilic or slightly basophilic vacuolated material found among the collagen fibers. They are usually found in the stroma of almost all types of invading tissue: carcinomas, sarcomas, and aggressive fibromatosis; within the stroma of metastatic tissue; in

TABLE 2. Cases with Negative Axillary Nodes

\begin{tabular}{crc}
\hline Variables & Count & Percent \\
\hline TG & 125 & \\
1 & 79 & 41.54 \\
2 & 96 & 26.2 \\
3 & & 31.8 \\
SMC & 195 & 64.7 \\
- & 106 & 35.2 \\
+ & & \\
Age & 95 & 31.5 \\
$<45$ & 71 & 23.5 \\
$46-55$ & 89 & 29.5 \\
$56-65$ & 46 & 15.2 \\
$>66$ & & \\
Size & 20 & 6.6 \\
T1b & 190 & 63.1 \\
T1c & 91 & 30.2 \\
T2 & & \\
Follow up & 249 & 11.6 \\
Alive & 35 & 5.6 \\
Reccurr & 17 & 9.9 \\
Died & 30 & \\
Censored & &
\end{tabular}


the periductal zone of high-grade intraductal carcinomas; and frequently within fibrous areas. It is important to differentiate mucoid stromal infiltration from stromal myxoid changes. The stroma around the mucin leakage is devoid of desmoplastic reaction, whereas stromal myxoid is usually intermingled with collagen and elastic fibers as well as lymphocytes, young fibroblasts, and endothelial cells.

\section{Grading Stromal Myxoid Changes}

Almost all tumors presented stromal myxoid changes but as a gradient of changes. Therefore, they were evaluated in three grades. Cases were recorded as absent or scanty (Grade 1), as moderate if at least three foci were found at the periphery (Grade 2), and as having marked stromal myxoid changes (Grade 3) when large areas of stromal myxoid changes were observed at the periphery and center of the tumor. They were assessed with a low-power objective $(10 \times)$ covering both the periphery and center of the lesion. At least two sections were evaluated in each case.

Because of the lower number of cases in the set for prognostic assessment, stromal myxoid changes were categorized as negative (Grade 1) or positive (Grades 2 and 3) according to the number of spots found, as explained above.

\section{Histological Evaluation for the Presence of Hyaluronan in the Stroma Reactions}

A biotinylated probe (a kind gift from Dr. C. Underhill at Georgetown University, Washington, DC) made up of a proteoglycan core protein that binds directly to hyaluronan was used to stain the tumor sections. The method of staining has been explained elsewhere (17). Briefly, after deparaffinization and hydration, endogenous peroxidase activity was suppressed by incubation in $10 \%$ hydrogen peroxidase in methanol for 10 minutes. The probe was dissolved in $10 \%$ fetal bovine serum in calcium and magnesium free phosphate buffered saline at a concentration of 1 to $2 \mu \mathrm{g} / \mathrm{mL}$. The tissue sections were incubated with the primary probe for 1 hour at $25^{\circ} \mathrm{C}$ and then for 15 minutes with a 1:500 dilution of peroxidase-conjugated streptavidin (Biogenex, CA). Thereafter, the sections were incubated for 30 minutes with 3-amino-9-ethil carbazole, counterstained with hematoxylin and eosin or ethyl green, and then mounted in glycerol. Between each incubation, the sections were washed with CMF-PBS twice, for 5 minutes, at $25^{\circ} \mathrm{C}$. The specificity of hyaluronan staining was determined with control sections treated for 24 hours at $37^{\circ} \mathrm{C}$ with 1500 TRU/dl Streptomyces hyaluronidase (Merck, Sharp y Dhom, NJ) to destroy hyaluronan before incubation with the probe.

\section{Quantification of Hyaluronan Staining}

Forty-six cases were semiquantified for hyaluronanstained areas. An image analysis system with the CAS 200 microscope and a morphometric soft (Micrometer-Analysis Systems) was employed as follows: tumor sections were stained with the hyaluronan probe according to the method explained above and counterstained with ethyl green. There are different optical thresholds for both stains. Each case was scanned with a $100 \times$ power objective in eight fields per case. Fields were selected searching for the hyaluronan-stained areas. The program selects and traces a line around areas stained with the probe (higher optical threshold, white spots), with the remaining, nonstained areas (ethyl green stained tissue with lower optical threshold) standing out as a gray background. Any field has an area ratio of stained (white areas) versus nonstained areas (gray areas). A final area ratio was obtained after average 8 fields (Fig. 1C).

\section{Conventional Prognostic Factors}

Greatest care was taken in the evaluation of the factors listed below. Cases were studied by four pathologists (MW, LCP, DC, VGD) who were blind to axillary status or prognosis; in case of disagreement, a final consensus was established. The validity and reproducibility of most variables were well established (18).

To organize a table for statistical analysis, we semiquantified stromal and tumor components according to 10 parameters and recorded them as categorical values (for example: 0 = absent, $1=$ scanty, $2=$ moderate, and $3=$ marked or diffuse). The following stromal changes were semiquantified.

Variable 1: stromal mixoid changes. The visual classification procedure was described above.

Variable 2: desmoplasia. The connective tissue replacement of the injured tissue resulting in a scar similar to skin scars is called desmoplasia. When there is a well-formed central sclerotic nodule often with acellular hyalinized keloid like tissue, this is considered to be marked desmoplasia (Grade 3). Lesser degrees of desmoplastic stromal reaction were semiquantified as scanty or absent (Grade 1) and intermediate (Grade 2).

Variable 3: tumor-infiltrating lymphocytes. They were evaluated as negative (Grade 1) when occasionally inflammatory cells were found and as moderate (Grade 2) and dense cellular (Grade 3) when this infiltration was found in the stroma surrounding tumor nests or inside tumor masses. 
Variable 4: tumor grade. This was evaluated based on the Scarff Bloom Richardson grading. The method (19) evaluates the following three parameters: degree of tubule formation, nuclear grade, and mitotic rate. Points are assigned to each parameter, and the histological grade is determined by summing up the points: Grade I, 1 to 5 points; Grade II, 6 to 7 points; Grade III, 8 to 9 points.

Variable 5: lymphatic embolization. This refers to peritumoral angiolymphatic invasion. If tumor cells involve peritumoral vascular spaces or are located beneath the skin, this is recorded as positive $=1$; otherwise, it is recorded as negative $=0$.

Variable 6: Tumor size. This variable is listed as categorical codes. Size of the tumor: less than $1 \mathrm{~cm}$ $=1 ; 1$ to $2 \mathrm{~cm}:=2$; more than $2 \mathrm{~cm}=3$.

Variable 7: age. We found that subdivision of cases into three age groups gave a better correlation with stromal myxoid changes than did a subdivision in two age groups, of pre- or postmenopausal status. Therefore, we identified a group of younger patients (aged $<45 \mathrm{y}$ ), recorded as 1 ; an intermediate group (aged 45 to 65 y) recorded as 2; and a group of older patients (aged $>65 \mathrm{y}$ ), recorded as 3 .

Variable 8: tumor borders. These were rated as follows: circumscribed $=1$, intermediate $=2$, and infiltrative $=3$.

Variable 9: multifocality. The tumor lesions were recorded as 1 if present and 0 if absent.

Variable 10: axillary status. Cases with negative axillary nodes were listed as 0 , and those with positive nodes were listed as 1 .

\section{Statistical Analysis}

Statistical analysis was performed by using the STATA computer program package. Frequency tables were employed for descriptive analysis. We used the $\chi^{2}$ test to estimate the association of axillary node status with categorical variables and the Mann Whitney $U$ test for continuous variables.

Once significant variables were identified, interactive stepwise logistic regression was used to build the multivariable model to estimate the effect of stromal myxoid changes on axillary node adjusting for possible confounders. For survival analysis, stromal myxoid changes were dichotomized (moderate or severe versus mild or none). We then used the log rank test to compare survival curves and Cox regression to estimate the hazard ratio of stromal myxoid changes to death.

\section{RESULTS}

\section{Histological Evaluation}

The stromal myxoid changes areas were usually at the peripheral zones but also could be found at the central zone of the tumor mainly in stromal myxoid changes Grade 3 (Fig. 3). Hyaluronan was strongly marked in the myxoid areas. It was also present in the desmoplastic areas, but its staining was reduced compared with the case of the myxoid changes. Inside desmoplasia, hyaluronan stains as faint filaments, whereas the myxoid areas were strongly marked with a combination of diffuse and thick filamentous staining (Fig. 1B). None of the control slides preincubated with Streptomyces hyaluronidase showed staining, confirming that the biotinylated probe was specifically linked to hyaluronan. Stromal myxoid changes Grade 3 usually have lymphatic embolization outside the tumor margin, and tumor- infiltrating lymphocytes were almost always found within the myxoid areas. Breast tumor cases with different degrees of stromal myxoid changes are exemplified in Figures 2 and 3.

\section{Semiquantification of Hyaluronan}

See Figure 4. Out of the 46 cases selected, 20 were categorized as negative stromal myxoid changes (Column A). The remainder 26 were categorized as positive stromal myxoid changes (Column B). There are highly significant differences in the mean area ratio from those categorized as stromal myxoid changes Grade 1 with median weak signal versus cases graded as stromal myxoid changes Grades 2-3 with median strong signal.

\section{Degree of Reproducibility of Stromal Myxoid Changes}

See Table 3. The stromal myxoid changes Grade 1 showed total agreement among two observers (LP, MMLP) and small variation in the third (AAM), resulting in substantial agreement among observers (kappa 0.77). Greater variations were observed in stromal myxoid changes Grade 2 (kappa: 0.53) and stromal myxoid changes Grade 3 (kappa $=0.69$ ). Overall kappa showed a substantial level of agreement (kappa: 0.67).

\section{Logistic Regression Analysis}

See Table 4. A multiple logistic regression analysis showed that positive nodes were related to stromal myxoid changes Grade 2 (odds ratio: 1.9) and stromal myxoid changes Grade 3 (odds ratio: 4.2), adjusting for the effect of desmoplasia, size, tumor infiltrating lymphocytes, tumor grade, lymphatic emboli, and multifocality.

\section{Cross Tabulation Studies of Stromal Myxoid Changes and Selected Variables}

See Table 5 for details of this section. 

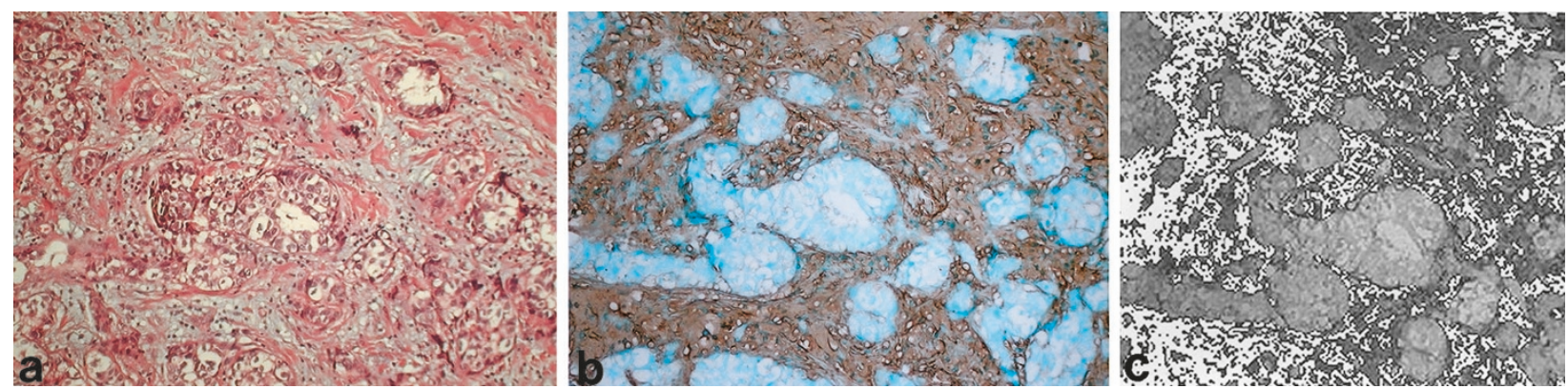

FIGURE 1. A, invading tumor nests and cords with stromal myxoid changes. Hematoxylin and eosin stain. B, consecutive section immunomarked with the hyaluronan probe. Counterstained with ethyl green. C, image analysis of the stromal hyaluronan signal. Midium power field (100 $\times)$. The program sums the white areas (immunomarked hyaluronan) and establishes an area ratio per field.
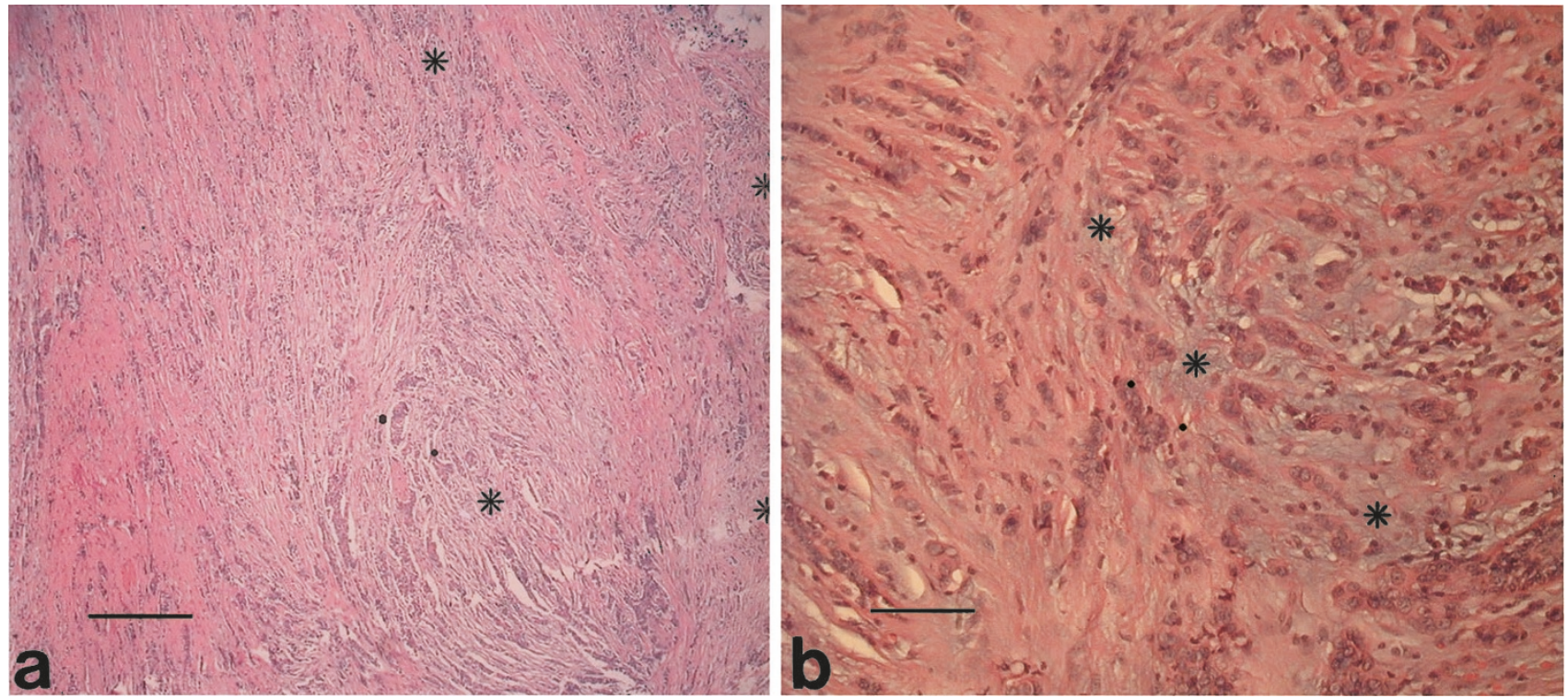

FIGURE 2. A, low-power field showing indian files pattern with myxoid changes of Grade 2. Asterisks indicate the stromal changes. These areas are usually regarded as edematous stroma. B, higher magnification showing files and thin strands with myxoid foci (asterisks) and desmoplastic stroma.
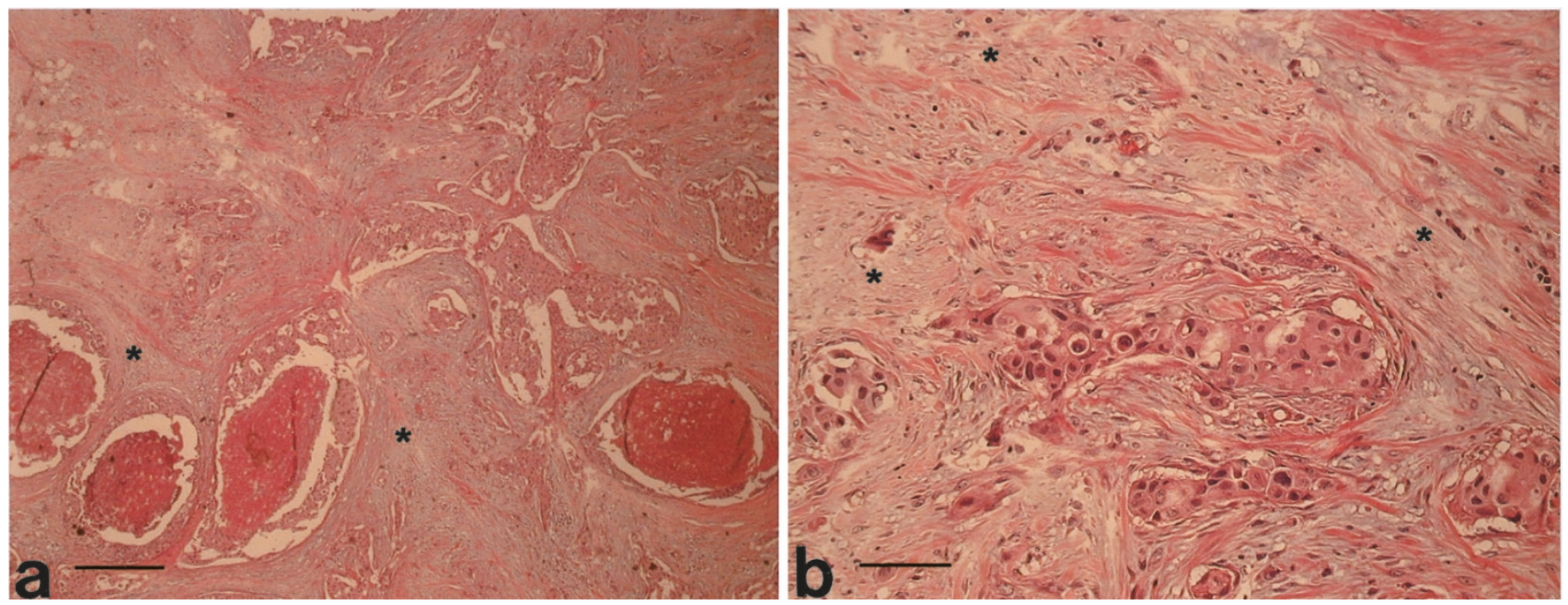

FIGURE 3. A, low-power field showing massive stromal changes (asterisks) graded as 3. B, from Panel A, higher magnification of upper left corner showing invading carcinoma cells with a myxoid stromal response. 


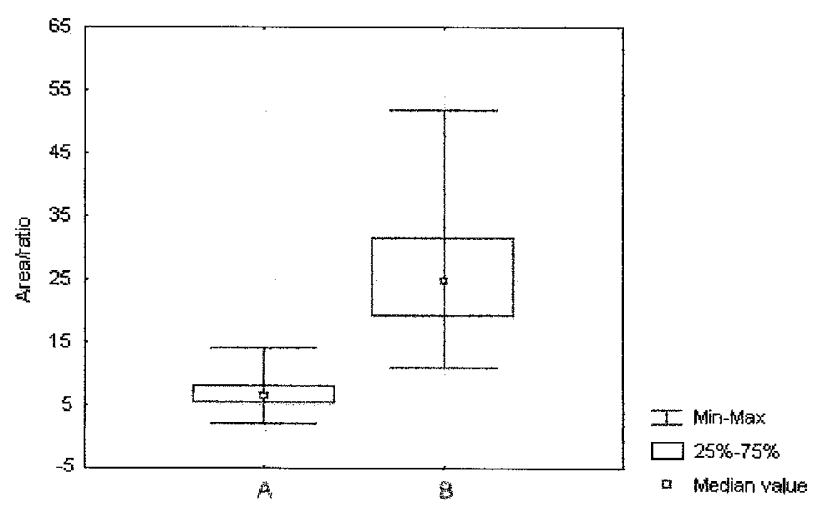

FIGURE 4. Boxplot comparing the mean intensity of HA stromal signal. Area ratio recorded by image analysis of 46 cases. A, n:20; SMC 1 ; area ratio ranging from 2 to 11 with a medium value of 5.5 . $\mathbf{B}, \mathrm{n}: 26$; SMC 2-3; area ratio ranging from 12 up to 55 with a medium value of $26(P<.000001$-Mann Whitney test $)$.

\section{Axillary Status}

The figures show that stromal myxoid changes Grade 1 (scanty), Grade 2 (focal), and Grade 3 (diffuse) have $23 \%, 33 \%$, and $43 \%$ of axillary metastasis respectively $(P<.001)$.

Age

Diffuse stromal myxoid changes (Grade 3) are significantly increased in patients aged $<45$ years. Within this subset, the proportion of Stromal Myxoid Changes 3 was $43 \%$, versus $24 \%$ and $20 \%$, found in older groups $(P<.001)$.

\section{Tumor Grade}

High Tumor Grade 3 shows greater frequency of diffuse Grade 3 stromal myxoid changes: $40 \%$, versus $24 \%$ for Grade 2 and $15 \%$ for Grade $1(P<.001)$.

TABLE 3. Reproducibility of Stromal Myxoid Changes

\begin{tabular}{lrrrrc}
\hline & \multicolumn{1}{c}{ LP } & \multicolumn{1}{c}{ AAM } & MMLP & KAPPA & Z \\
\hline SMC 1 & $114(56.4)$ & $111(54.9)$ & $114(56.4)$ & 0.7791 & 19.18 \\
SMC 2 & $52(25.7)$ & $57(28.2)$ & $60(29.7)$ & 0.5323 & 13.10 \\
SMC 3 & $36(17.8)$ & $34(16.8)$ & $28(13.8)$ & 0.6957 & 17.13 \\
Combined & & & & 0.6746 & 22.29 \\
\hline
\end{tabular}

TABLE 4. Logistic Regression Analysis with Axillary Status as the Dependent Variable

\begin{tabular}{lccccc}
\hline & Odds Ratio & Interval & $95 \%$ Conf & $\mathrm{z}$ & $P$ \\
\hline SMC 2 & 1.96 & 2.83 & 1.3 & 3.4 & .000 \\
SMC 3 & 4.22 & 6.53 & 2.7 & 6.4 & .000 \\
Emboli & 1.7 & 2.97 & 0.97 & 1.8 & .063 \\
D 2 & 1.4 & 2.17 & 0.91 & 1.5 & .124 \\
D 3 & 3.4 & 5.36 & 2.1 & 5.2 & .000 \\
T1c & 3.2 & 6.21 & 1.67 & 3.5 & .000 \\
T2 & 4.6 & 9.10 & 2.40 & 4.5 & .000 \\
TG 2 & 2.5 & 3.79 & 1.72 & 4.6 & .000 \\
TG 3 & 7.0 & 11.09 & 4.46 & 8.3 & .000 \\
TIL 2 & 2.3 & 3.48 & 1.54 & 4.0 & .000 \\
TIL 3 & 1.5 & 2.92 & 0.83 & 1.3 & .135 \\
MTF & 1.5 & 2.24 & 1.09 & 2.4 & .013 \\
\hline
\end{tabular}

TABLE 5. Cross Tabulation of Stromal Myxoid Changes and Selected Variables

\begin{tabular}{|c|c|c|c|c|}
\hline Variables & $\mathrm{SMC}+$ & $\begin{array}{c}\text { SMC } \\
++\end{array}$ & $\begin{array}{l}\text { SMC } \\
+++\end{array}$ & $P$-level \\
\hline \multicolumn{5}{|l|}{ ALN } \\
\hline- & 361 (55) & $197(30)$ & 100 (15) & \\
\hline+ & 109 (23) & $154(33)$ & $201(43)$ & $\mathrm{x}: 141 ;<.000$ \\
\hline \multicolumn{5}{|l|}{ Size } \\
\hline Tla-b & $98(58)$ & $42(25)$ & $29(17)$ & \\
\hline Tlc & $202(43)$ & $155(33)$ & $113(24)$ & \\
\hline $\mathrm{T} 2$ & $168(35)$ & $149(31)$ & $156(33)$ & $\mathrm{x}: 33 ;<.000$ \\
\hline \multicolumn{5}{|l|}{ Age } \\
\hline$<45$ & $52(27)$ & $56(29)$ & $82(43)$ & \\
\hline $46-65$ & $202(43)$ & $168(33)$ & $120(24)$ & \\
\hline$>66$ & $153(49)$ & $93(30)$ & $63(20)$ & $\mathrm{x}: 40 ;<.000$ \\
\hline \multicolumn{5}{|l|}{ TG } \\
\hline 1 & $62(58)$ & $29(27)$ & 15 (15) & \\
\hline 2 & $259(41)$ & $254(36)$ & $161(22)$ & \\
\hline 3 & $119(38)$ & $68(21)$ & $125(40)$ & $\mathrm{x}: 52 ;<.000$ \\
\hline \multicolumn{5}{|l|}{ Emboli } \\
\hline- & $459(46)$ & $324(32)$ & $212(21)$ & \\
\hline+ & $11(8)$ & $27(21)$ & $89(70)$ & $\mathrm{x}: 132 ;<.000$ \\
\hline \multicolumn{5}{|l|}{ TIL } \\
\hline 1 & 352 (43) & $255(31)$ & $210(26)$ & \\
\hline 2 & $62(27)$ & $80(36)$ & 80 (36) & \\
\hline 3 & $56(67)$ & $16(19)$ & $11(14)$ & $\mathrm{x}: 42 ;<.000$ \\
\hline \multicolumn{5}{|l|}{ Desmoplasia } \\
\hline 1 & $108(44)$ & 44 (18) & $93(37)$ & \\
\hline 2 & 163 (39) & 139 (33) & $116(28)$ & \\
\hline 3 & $199(43)$ & $168(36)$ & $92(29)$ & $\mathrm{x}: 41 ;<.000$ \\
\hline
\end{tabular}

\section{Lymphatic Emboli}

Of cases with positive lymphatic emboli, $70 \%$ have diffuse Grade 3 stromal myxoid changes $(P<.001)$.

\section{Tumor-Infiltrating Lymphocytes and Desmoplasia}

These are inversely related to stromal myxoid changes.

\section{Stromal Myxoid Changes as a Prognostic Factor of Mortality}

Among 301 patients free of axillary metastases, those showing stromal myxoid changes of Grade 2 or 3 had an increased risk of death over the 10-year follow-up period (log rank test, $P<.01$, hazard ratio $3.05,95 \%$ confidence interval [1.2-7.9]). There is a stronger prognostic effect within the T2 cases (Fig. 5), but it is a subset too small for clear conclusions. Using Cox regression analysis to adjust for age, tumor size, and tumor grade, stromal myxoid changes Grades 2 or 3 showed a hazard ratio of 2.21 confidence interval 95\% (0.87-5.2), $P=$ NS (data not shown). The wide confidence interval is probably caused by the low death rate within the cohort (17 of 301 patients) in 10 years of follow up. Therefore the multivariate test does not validate stromal myxoid changes as an independent prognostic factor.

\section{DISCUSSION}

In this study, it was found that stromal changes seen in breast carcinoma are associated with ad- 
verse parameters such as high histological grade, emboli, young age, and the presence of axillary metastases. Therefore, the presence of stromal changes helps identify high-risk groups.

Visual impressions of stromal myxoid changes were compared with quantitative morphometry. The results indicate that morphometric measurements of stromal signals show high correlation with the visual evaluation, confirming that the histological assessment is as reliable as the digital imaging techniques.

The reproducibility of the stromal myxoid changes indicates that interobserver agreement can be achieved in a substantial number of cases. Discrepancies among pathologists were mainly confined to the intermediate group. However, there was an acceptable level of reproducibility even in this group.

With the multivariate analysis, a strong relationship of stromal myxoid changes to positive axillary nodes was found even after adjusting them for important confounders. An independent association of tumor grade was observed, but no relationship was found between Grade 1 and positive nodes (data not shown). De La Torre et al. (20) did not find any relationship between stromal changes and nodal metastasis in tubular carcinoma. We support his findings given the fact that Grade 1 tumors (all tubular carcinomas are Grade 1) did not correlate with positive nodes. However, a strong relationship does appear between myxoid changes with Tumor Grades 2 and 3 and positive nodes. Thus, multivariate analysis indicates that there is a synergistic effect of tumor grade, tumor size, and stromal myxoid changes with the metastatic process.

As was mentioned before, hyaluronan synthesis is stimulated by the interaction between tumor and stromal cells, hence stromal myxoid changes could be the expression of an early primitive response to the invading carcinoma. But stromal myxoid changes do not necessarily indicate a parameter of tumor aggressiveness. Stromal myxoid changes should be interpreted as a noncellular stromal response occurring in any situation that causes stromal injury, facilitating tumor, or non-tumor cell migration. The negative association with desmoplasia and tumor lymphocytic infiltration in the cross tabulation and multivariate analysis study suggests fibrous and cellular replacement. Probably, stromal myxoid changes provide a provisional matrix for a granulation tissue as a response and are precursors of desmoplasia. Three lines of evidence support such a proposal. First, hyaluronan was found to be the ground substance for migration of lymphocytes as it can synergistically act with T-cell lymphocytes and secrete factors that promote vessel migration as part of an inflammatory response (21-23). Second, hyaluronan plays a well-known role in the molecular sequence leading to collagen fibrillogenesis (24). Third, extracellular matrix hyaluronan is prominent during wound healing, appearing at elevated levels early in the repair process. The degradation of hyaluronic acid results in increased fibroplasia, collagen deposition, and keloid tissue (25). Presumably, if there was some impairment in the production or functionality of hyaluronan, granulation tissue, and subsequent desmoplasia would not develop, and the enzymatic metastatic cascade might be delayed or blocked.

Another finding was that young age appears as an important factor in the induction of stromal myxoid changes. Several observation could imply that steroids are possibly stimulators of the stromal myxoid changes: increased hyaluronan biosynthesis accompanies decidual responses in the endometrium (26), maternal serum hyaluronic acid concentrations increase as pregnancy progresses and serum levels increase significantly at term (27), hyaluronic acid is associated with cervical ripening during parturition (28), and marked stromal myxoid changes have been reported to be under a strong progestational effect in rectal endometriosis (29). All these reports suggest that the ovarian hormones play an important role in modulating hyaluronic acid synthesis and could provide an explanation of the association of stromal myxoid changes with young age.

In addition, a strong association of stromal myxoid changes with lymphatic embolizations was found. The importance of this observation is exemplified by the fact that clinicopathological findings have long suggested that by providing a pathway for tumor cell dissemination, lymphatics are a key component of metastatic spread. It is also well known that the lymphatic system is the main organ for the transporting and turnover of hyaluronan from tissues. Receptor-mediated transcytosis of hyaluronan may contribute to tumor cell transporting by paracellular pathways. Jackson et al. (30) speculate that carcinoma cells invade the lymphatic

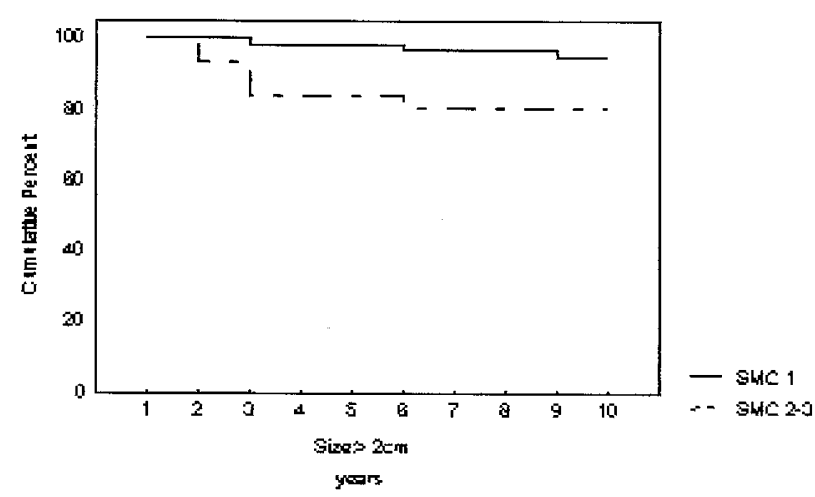

FIGURE 5. Tumor size larger than $2 \mathrm{~cm}$. Sixty cases with - SMC. Within this group there were 3 deaths versus 31 cases with + SMC and 6 deaths $(P<.008)$. 
vessels using hyaluronan as a molecular link between CD44/hyaluronate-receptor-bearing tumor cells and LYVE-1/hyaluronate receptor-lymphatic endothelial receptors giving rise to lymphatic embolizations .

The increased mortality in patients having positive stromal myxoid changes is consistent with these findings. Nevertheless, although statistically significant, the multivariate analysis differences are not statistically significant. Auvinen et al. (11), using a similar histopathological evaluation, conclude that the intensity of the stromal hyaluronan signal and the presence of cells associated with hyaluronan were both significantly related to poor differentiation of the tumors, axillary lymph node positivity, and overall shorter survival of the patients. They propose that hyaluronan disrupts the extracellular matrix, creating invasionpermissive conditions. In general terms, we agree with their findings; however, we cannot confirm in our series of patients that myxoid changes are an independent prognostic factor. As mentioned in the Materials and Methods section, 54 patients with highgrade tumors were selected for adjuvant chemotherapy. In a previous publication (31), we found 19 deaths over 5 years $(11.5 \%)$ in a cohort of 165 cases with negative nodes and without adjuvant chemotherapy. There are obvious differences in the mortality within the current cohort, with 17 deaths at 10 years $(5.6 \%)$. Patients with high-grade tumors who died in our previous and current series are more frequently associated with stromal myxoid changes. Selection of aggressive cases for adjuvant chemotherapy might have distorted the survival curves. Further research based on untreated patients is needed to establish the clinical significance of these findings.

It could be hypothesized that stromal myxoid changes conform to a matrix full of survival and motility factors for vessels and mononuclear and neoplastic cells that otherwise would be cleared by apoptosis. Eventually, this matrix could be used for diagnostic or therapeutic purposes. Hyaluronan could be employed as an adherent net for liposomes containing contrast agents for magnetic resonance visualization $(32,33)$. Moreover, extracellular hyaluronan can facilitate cellular uptakes of properly designed microvesicles $(34,35)$. Conceivably, liposome-mediated plasmid transfection could engineer the cancer cell genome, inducing the synthesis of peptides to block in situ the function of hyaluronan (36). Targeting hyaluronan could arrest multiple biological pathways and might be used as a strategy to improve the treatment of cancer.

Acknowledgments: The authors are indebted to Kevin Mc Carthy, Bryan P. Toole, Juan Rosai, and Andrea Herrera-Gayol for reviewing the manuscript and giving helpful advice, to Esteban Mocetti for technical support, and to Fernando Rubinstein for the statistical study. We especially remember the late Juan Carlos Lamattina for follow-up of patients, carefully obtained over the course of his productive life, and the late Alicia Perez Companc for her generous support. Both were of paramount importance to our investigation.

\section{REFERENCES}

1. Brown CT, Applebaum E, Banwatt R, Trinkaus-Randall V. Synthesis of stromal glycosaminoglycans in response to injury. J Cell Biochem 1995;59:57-68.

2. Knudson W, Biswas C, Toole BP. Interactions between human tumor cells and fibroblasts stimulate hyaluronate synthesis. Proc Natl Acad Sci U S A 1984;81:6767-71.

3. Suzuki M, Asplund T, Yamashita H, Heldin CH, Heldin P. Stimulation of hyaluronan biosynthesis by platelet-derived growth factor-BB and transforming growth factor-beta 1 involves activation of protein kinase. Biochem J 1995;307:817-21.

4. Fraser JR, Laurent TC, Laurent UB. Hyaluronan. Its nature, distribution, functions and turnover. J Intern Med 1997;242: 27-33.

5. Toole BP, Biswas C, Gross J. Hyaluronate and invasiveness of the rabbit V2 carcinoma. Proc Natl Acad Sci U S A 1979;12: 6299-303.

6. Bartolazzi A, Peach R, Aruffo A, Stamenkovic I. Interaction between CD44 and hyaluronate is directly implicated in the regulation of tumor development. J Exp Med 1994;180:53.

7. Goebeler M, Kaufmann D, Brocker EB, Klein CE. Migration of highly aggressive melanoma cells on hyaluronic acid is associated with functional changes, increased turnover and shedding of CD44 receptors. J Cell Sci 1996;109:1957-64.

8. Zhang L, Underhill CB, Chen L. Hyaluronan on the surface of tumour cells is correlated with metastatic behavior. Cancer Res 1995;55:428-33.

9. Ropponen KM, Tammi J, Parkkinen J, Eskelinen M, Tammi $\mathrm{R}$, Lipponen $\mathrm{P}$, et al. Tumor cell hyaluronan as an unfavorable prognostic factor in colorectal cancer. Cancer Res 1998; 58:342-7.

10. Ambros RA, Malfetano JH, Mihm MC. Clinicopathologic features of vulvar squamous cell carcinomas exhibiting prominent fibromyxoid stromal response. Int J Gynecol Pathol 1996;15:137-45.

11. Auvinen P, Tammi RH, Parkkinen J, Tammi M, Johansson RT, Agren UM, et al. Hyaluronan in peritumoral stroma and malignant cells associates with breast cancer spreading and predicts survival. Am J Pathol 2000;156:529-36.

12. Bertrand P, Girard N, Delpech B. Hyaluronan (hyaluronicacid) and hyaluronectin in the extracellular-matrix of human breast carcinomas-comparison between invasive and noninvasive areas. Int J Cancer 1992;52:1-6.

13. Setälä LP, Tammi MI, Eskelinen MJ, Lipponen PK, Agren UM, Parkkinen J, et al. Hyaluronan expression is associated with local and nodal spread and reduced survival rate. Br J Cancer 1999;79:1133-8.

14. Anttila MA, Tammi RH, Tammi MI, Syrjänen KJ, Saarikoski SV, Kosma VK. High levels of stromal hyaluronan predict poor disease outcome in epithelial ovarian cancer. Cancer Res 2000;60:150-5.

15. Landis JR, Koch GG. The measurement of observer agreement for categorical data. Biometrics 1977;33:159-74.

16. Arrighi A, Lamattina JC, Wernicke M, Guixa H, Loruso C, Orti R. Breast carcinoma with node negative and high aggressiveness. Value of adjuvant chemotherapy. Rev Hosp Ital Buenos Aires 1992;2:16-9. 
17. Azurri N, Underhill CB, Kagan E, Banni KS. A novel biotinylated probe specific for hyaluronate. Am J Surg Pathol 1992; 16:116-21.

18. Association of Directors of Anatomic and Surgical Pathology. Recommendations for the reporting of breast cancer. Mod Pathol 1996;9:77-81.

19. Elston CW, Ellis IO. Pathological prognostic factors in breast carcinoma. The value of histological grade in human breast carcinoma. Experience from a large study with long-term follow up. Histopathology 1991;19:403-10.

20. De La Torre M, Wells AF, Bergh J, Lindgren A. Localization of hyaluronan in normal breast radual scar tissue and tubular breast carcinoma. Hum Pathol 1993;24:1294-7.

21. Mohamadzadeh M, DeGrendele H, Arizpe H, Estess $P$, Sieguelman M. Proinflammatory stimuli regulate endothelial hyaluronan expression and CD44/HA-dependent primary adhesion. J Clin Invest 1998;101:97-108.

22. DeGrendele HC, Estess P, Siegelman MH. Requirement for CD44 in activated $\mathrm{T}$ cell extravasation into inflammatory site. Science 1997;278:672-5.

23. Freeman MR, Schneck FX, Gagnon ML, Corless C, Soker S, Niknejad K, et al. Peripheral blood T lymphocytes and lymphocytes infiltrating human cancers express vascular endothelial growth factor: a potential role for T cells in angiogenesis. Cancer Res 1995;55:4140-5.

24. Ellis IR, Schor SL. Differential effects of TGF-betal on hyaluronan synthesis by fetal and adult skin fibroblasts: implications for cell migration and wound healing. Exp Cell Res 1996;228:326-33.

25. Meyer LJ, Russell SB, Russell JD, Trupin JS, Egbert BM, Shuster $\mathrm{S}$, et al. Reduced hyaluronan in keloid tissue and cultured keloid fibroblasts. J Invest Dermatol 2000;114:953-9.

26. Carson DD, Dutt A, Tang JP. Glycoconjugate synthesis during early pregnancy: hyaluronate synthesis and function. Dev Biol 1987;120:228-35.
27. Kobayashi H, Sun GW, Tanaka Y, Kondo T, Terao T. Serum hyaluronic acid levels during pregnancy and labor. Obstet Gynecol 1999;93:480-4.

28. Obara M, Hirano H, Ogawa M, Tsubaki H, Hosoya N, Yoshida Y, et al. Changes in molecular weight of hyaluronan and hyaluronidase activity in uterine cervical mucus in cervical ripening. Acta Obstet Gynecol Scand 2001;80:492-6.

29. McCluggage WG, Kirk SJ. Pregnancy associated endometriosis with pronounced stromal myxoid change. J Clin Pathol 2000;53:241-2.

30. Jackson DG, Prevo R, Clasper S, Banerji S. LYVE-1, the lymphatic system and tumor lymphangiogenesis. Trends Immunol 2001;22:317-21.

31. Wernicke M, Podesta A. Evaluation of breast carcinoma according with a scoring method. J Surg Oncol 1982;21:107-13.

32. Pasquali-Ronchetti I, Quaglino D, Mori G, Bacchelli B, Ghosh P. Hyaluronan-phospholipid interactions. J Struct Biol 1997;120:1-10.

33. Grunder W, Biesold M, Wagner M, Werner A. Improved nuclear magnetic resonance microscopic visualization of joint cartilage using liposome entrapped contrast agents. Invest Radiol 1998;33:193-202.

34. Ruponen M, Ronkko S, Honkakoski P, Pelkonen J, Tammi M, Urtti A. Extracellular glycosaminoglycans modify cellular trafficking of lipoplexes and polyplexes. J Biol Chem 2001; 276:33875-80.

35. Eliaz RE, Szoka FC Jr. Liposome-encapsulated doxorubicin targeted to CD44: a strategy to kill CD44-overexpressing tumor cells. Cancer Res 2001;61:2592-601.

36. Mummert ME, Mohamadzadeh M, Mummert D, Mizumoto N, Takashima A. Development of a peptide inhibitor of hyaluronan-mediated leukocyte trafficking. J Exp Med 2000; 192:769-80. 